\title{
Lung Ultrasonography in Diagnosis and Management of Novel Coronavirus (COVID-19) Pneumonia: Pearls and Pitfalls
}

\author{
Heng Xue, MD ${ }^{a}$, Yao Zhang, MD ${ }^{b}$, Ligang Cui, MD ${ }^{a, *}$, Jing Han, MD ${ }^{c}$ \\ ${ }^{a}$ Department of Ultrasound, Peking University Third Hospital, Beijing, China; ${ }^{b}$ Department of Ultrasound, Beijing Ditan Hospital, \\ Capital Medical University, Beijing, China; ${ }^{c}$ Ultrasound and Functional Diagnostic Center, Beijing You An Hospital, Capital Medical \\ University, Beijing, China \\ Received April 08, 2020; revision received April 10, 2020; accepted April 11, 2020.
}

\begin{abstract}
The purpose of this article is to review the use of lung ultrasonography (US) in the workup of COVID-19 pneumonia. The scanning protocol, normal US appearance of lung, major US features of COVID-19 pneumonia, diagnostic performance of lung US and potential pitfalls when explaining US results are descripted and discussed. Lung US is increasingly accepted as a useful tool in the workup of COVID-19 pneumonia. Certain US imaging features allow to confirm or rule out the diagnosis for clinical management; on other hand, most US findings are nonspecific with technical limitations. Thus, it is important to recognize these drawbacks since the ignorance of potential pitfalls of lung US may lead to over diagnosis or missed diagnosis.
\end{abstract}

Key words: Lung ultrasonography; COVID-19 pneumonia; Technical limitation; Pitfalls

Advanced Ultrasound in Diagnosis and Therapy 2020;02:057-059

DOI: 10.37015/AUDT.2020.200030

$\mathrm{T}$ he utilization of lung US has been greatly developed with technological development in the last decades. In addition to the study of pleural effusion, its main applications include triage the acute respiratory failure in the emergency and monitoring of pulmonary congestion/reaeration in critical care settings. As reported by recent studies, this technique has been used as an important modality in the early diagnosis and management of COVID-19 pneumonia [1,2], which evolved into a pandemic worldwide. Besides its advantages of safety, repeatability, low cost and pointof-care, it could reduce the risk for transporting unstable patients to $\mathrm{CT}$ rooms and minimize the exposure risk for medical devices and health-care workers [3]. This is especially important to prevent nosocomial outbreaks considering the high contagiousness of the virus.

\section{Scanning Technique and Normal US Appearance of Lung \\ Preventing contamination and infection is critical}

during medical services and patient care at the outbreak of COVID-19 epidemics. Before scanning patients with suspected COVID-19, clinicians should wear personal protective equipment according to WHO [4]. US transducer and equipment should be cleaned and disinfected before and after scanning as per guidelines in prevention of virus transmission $[5,6]$.

The lung US can be performed for patients in sitting, supine or decubitus position. Each intercostal space available should be assessed. In order to facilitate comparative assessment of lesions in the following exams, the bilateral lung could be further divided into anterior superior, anterior inferior, lateral superior, lateral inferior, and posterolateral fields. Convex probes (2$5 \mathrm{MHz})$, which have better penetration and larger fieldof-view, should be used first to get a general impression of the patient's lung. Linear probe $(3-9 \mathrm{MHz})$ could be further used in uncertain area to demonstrate more details in the lung surface. Color Doppler imaging is not routinely used unless consolidation lesion appears.

\footnotetext{
* Corresponding author: Department of Ultrasound, Peking University Third Hospital, 49 Northe Garden Rd, Haidian District, Beijing, China e-mail: ligangcui@pku.edu.cn 
The normal aerated lung surface coved by two layers of the pleura is visualized as curvilinear hyperechoic interface with posterior echogenic parallel horizontal lines. The surface hyperechoic line is called pleural line and these posterior echogenic lines coursed by reverberation artifact are called A lines.

\section{US Features of COVID-19 Pneumonia}

COVID-19 pneumonia typically involves peripheral, posterior zone of lower lung [7-9]. The number of studies focusing on lung US features for COVID-19 pneumonia is limited $[1,2]$. US findings of the disease were summarized based upon previous studies and our experiences: (1). Multifocal, discrete, or confluent B lines, which were multiple vertical echogenic reverberation artifacts extending from the lung surface without attenuation. This is caused by reverberation of the ultrasound beam between the slightly decreased alveolar air and increased interstitial fluids. Multiple focal B lines $(\geq 3)$ usually appear in the early stage or mild type patients. Diffuse confluent B lines (waterfall sing) (Fig 1) in the following lung US studies indicate disease progression. On the contrary, during the recovery phase, A lines, which are multiple linear echogenic horizontal lines below the lung surface, would replace B lines. (2). Thickened pleural line. Similar to CT feature, this sign indicates pulmonary fibrosis. (3). Small patchy of consolidation, occasionally with air bronchograms. This visualized on US as tissue-like hypoechoic region (Fig 2), which reflects the pathological process of highly reduced air and increased inflammatory cellular exudate. (4). Pleural effusion. This is uncommon seen in COVID-19 pneumonia. These US features are not isolated but may appear with one to another. The US features and distributions are related to the stage and severity of the disease, therefore, the repeatability of lung US studies in critically ill patients with COVID-19 pneumonia guarantees the effect monitoring of antiviral and supportive therapies.

\section{Diagnostic Performance and Potential Pitfalls of Lung US}

In respect of diagnostic performance, lung US could provide results superior to chest radiography and similar to chest $\mathrm{CT}$ for evaluation of bacterial and viral pneumonia to acute respiratory distress syndrome (ARDS) [10,11]. The major drawback of lung US is that the beam cannot penetrate aerated lung. Therefore lesions not locating at or extending to the pleural surface are invisible on lung US. However, since $90 \%$ of COVID-19 pneumonia involve peripheral lungs [7-9], this drawback would not preclude the utility of lung US in patients suspected of COVID-19 pneumonia.

Recent studies reported that chest CT showed high
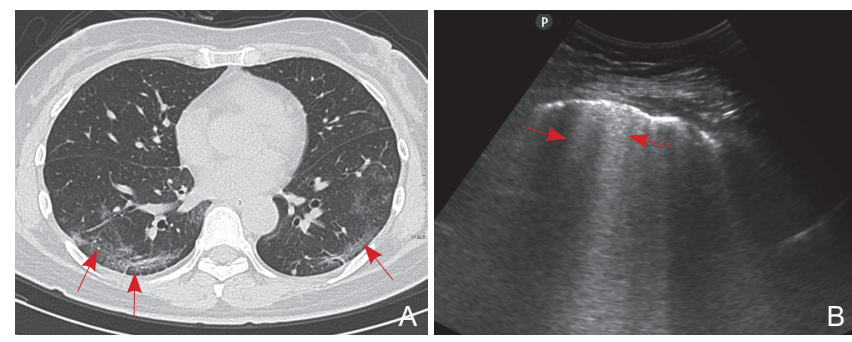

Figure 1 A 51-year-old female patient with an exposure history of traveling to Wuhan, presented with fever and cough for 9 days. (A) Chest CT shows multiple patchy ground grass opacities (GGO) (arrows) in the bilateral peripheral lung. (B) Lung US in the corresponding location shows confluent B lines (waterfall sign) (arrows).
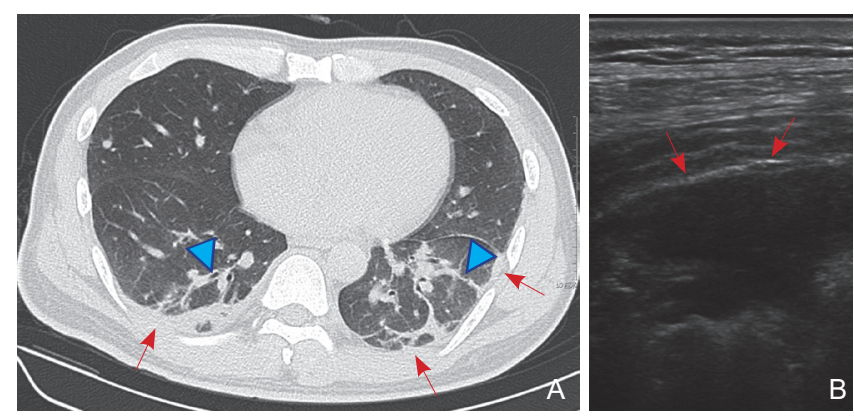

Figure 2 This 31-year-old male patient had close contact with COVID-19 pneumonia patient. He had fever for 1 day. (A) Chest CT shows multiple subpleural consolidation (arrows) and interlobular septal thickening (arrowheads) in the bilateral peripheral lung. (B) Lung US using high frequency linear probe in the corresponding location shows tissue-like hypoechoic region (arrows) representing a focal consolidation.

sensitivity in patients under emulation for COVID-19 although the specificity and positive predictive value were only modest [12-14]. Lung US has the same problem with chest CT. None of the lung US features could be specific to confirm the diagnosis of COVID-19 pneumonia. The major feature of multiple B-lines simply reflect increased interstitial fluids. Visualization of the B-line cannot differentiate between different etiologies as cardiogenic edema, ARDS, pulmonary fibrosis or viral pneumonia [15], and even $16 \%$ of the healthy volunteers had 2 or more B-lines [16]. Consequently, clinicians should be cautious when explaining "abnormal" results of lung US. Comprehensive evaluation of the patients, including clinical manifestation (fever and/or cough), blood test (decreased white blood cells), history of epidemic exposure, reverse transcription polymerase chain reaction should be completed before confirming or ruling out the diagnosis of COVID-19 pneumonia.

In some circumstances, lung US may not be as sensitive as chest $\mathrm{CT}$ even the lesions extend to the pleural surface. Here, we present two confirmed 
COVID-19 pneumonia cases with ground grass opacities (GGO) on CT but normal on lung US. In one case, US imaging with a convex probe was not able depict all subpleural GGO abnormalities on CT (Fig 3). In this situation, high frequency linear probe should be used to find more subtle changes. Unfortunately, the images were not obtained in this case. In another case, small patchy opacities seen on CT were obscured by rib (Fig 4).
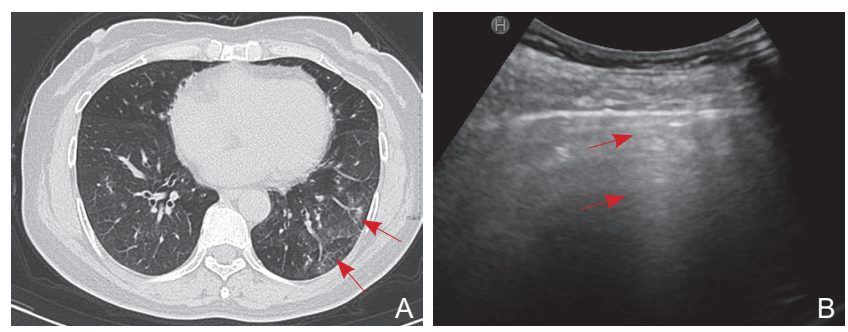

Figure 3 This 58-year-old female had sore throat for 6 days and fever for 3 days, and she had close contact with COVID-19 pneumonia patient. (A) Chest CT shows multiple patchy GGO (arrows) in the left peripheral lung. Lung US in the corresponding location reveals suspected B lines (arrows).
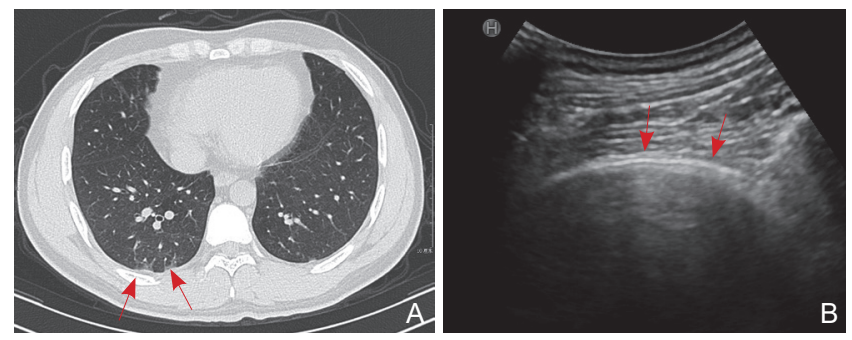

Figure 4 This 30-year-old male patient had fever and cough for 1 day. (A) Chest CT shows small patchy opacities (arrow) in the right subpleural area deep to the rib. (B) Lung US in the corresponding location reveals normal pleural line (arrow). The lesion might be obscured by coastal rib.

\section{Conclusion}

Lung US is being increasingly used as the initial and follow up imaging modality for evaluating various pulmonary pathologies, including COVID-19 pneumonia. Emphasis on proper scanning protocols, recognition of major lung US features, i.e., Pleural line, A-lines, B-lines, echogenic consolidation, pleural thickening, and effusion, are premises for accurate diagnosis and management of COVID-19 pneumonia. However, most pulmonary pathologies have nonspecific US findings, clinical information, epidemic exposure, laboratory test and chest $\mathrm{CT}$ should be comprehensively reviewed and considered before confirming or ruling out the diagnosis. Thus, it is important to recognize these limitations since the ignorance of potential pitfalls of lung US may lead to over diagnosis or missed diagnosis.

\section{Conflict of Interest}

The authors have no conflict of interest to declare.

\section{References}

[1] Poggiali E, Dacrema A, Bastoni D, Tinelli V, Demichele E, Mateo Ramos P, et al. Can lung US help critical care clinicians in the early diagnosis of novel coronavirus (COVID-19) pneumonia? Radiology 2020: 200847. DOI: 10.1148/radiol.2020200847.

[2] Peng QY, Wang XT, Zhang LN; Chinese Critical Care Ultrasound Study Group (CCUSG). Findings of lung ultrasonography of novel corona virus pneumonia during the 2019-2020 epidemic. Intensive Care Med. 2020: 1-2. DOI: 10.1007/s00134-020-05996-6.

[3] Buonsenso D, Pata D, Chiaretti A. COVID-19 outbreak: less stethoscope, more ultrasound. Lancet Respir Med. 2020 Mar 20. DOI: $10.1016 / \mathrm{S} 2213-2600(20) 30120-\mathrm{X}$.

[4] WHO. Medical devices. Personal protective equipment. 2020 Available from: https://www.who.int/medical_devices/meddev_ppe/ en/.

[5] Costello C, Basseal J, Yang Y, Anstey J, Yastrebov K. Prevention of pathogen transmission during ultrasound use in the Intensive Care Unit: Recommendations from the College of Intensive Care Medicine Ultrasound Special Interest Group (USIG). Australasian Journal of Ultrasound in Medicine.

[6] Abramowicz J, Basseal J. WFUMB Position Statement: How to perform a safe ultrasound examination and clean equipment in the context of COVID-19. World Federation for Ultrasound in Medicine and Biology (WFUMB). Available from: https://wfumb. info/2020/03/27/ss-covid19-001/

[7] Song F, Shi N, Shan F, Zhang Z, Shen J, Lu H, et al. Emerging 2019 novel coronavirus (2019-nCoV) pneumonia. Radiology 2020; 295: 210-217.

[8] Salehi S, Abedi A, Balakrishnan S, Gholamrezanezhad A. Coronavirus disease 2019 (COVID-19): a systematic review of imaging findings in 919 patients. AJR Am J Roentgenol 2020: 1-7.

[9] Bai HX, Hsieh B, Xiong Z, Halsey K, Choi JW, Tran TML, et al. Performance of radiologists in differentiating COVID-19 from viral pneumonia on chest CT. Radiology 2020: 200823. DOI: 10.1148/ radiol.2020200823.

[10] Mayo PH, Copetti R, Feller-Kopman D, Mathis G, Maury E, Mongodi S, et al. Thoracic ultrasonography: a narrative review. Intensive Care Med 2019; 45: 1200-1211

[11] Laursen CB, Sloth E, Lassen AT, Christensen Rd, Lambrechtsen J, Madsen PH, et al. Point-of-care ultrasonography in patients admitted with respiratory symptoms: a single-blind, randomised controlled trial. Lancet Respir Med 2014; 2: 638-46.

[12] Dai WC, Zhang HW, Yu J, Xu HJ, Chen H, Luo SP, et al. CT imaging and differential diagnosis of COVID-19. Can Assoc Radiol J 2020; 71: 195-200.

[13] Ai T, Yang Z, Hou H, Zhan C, Chen C, Lv W, et al. Correlation of chest CT and RT-PCR testing in coronavirus disease 2019 (COVID-19) in China: A Report of 1014 Cases. Radiology 2020: 200642. DOI: 10.1148/radiol.2020200642.

[14] Fang Y, Zhang H, Xie J, Lin M, Ying L, Pang P, et al. Sensitivity of Chest CT for COVID-19: Comparison to RT-PCR. Radiology 2020: 200432. DOI: 10.1148/radiol.2020200432.

[15] Volpicelli G. Lung sonography. J Ultrasound Med 2013; 32: 165-71.

[16] Zoneff ER, Baker K, Sweeny A, Keijzers G, Sanderson J, Watkins S. The prevalence of lung surface abnormalities in a healthy population as detected by a screening lung ultrasound protocol: Comparison between young and older volunteers. Australasian Journal of Ultrasound in Medicine 2019;22:129-37. 